\title{
Tabbed Tissue Expanders Improve Breast Symmetry Scores in Breast Reconstruction
}

\author{
Nima Khavanin, Madeleine J Gust, David W Grant, Khang T Nguyen, John YS Kim \\ Division of Plastic and Reconstructive Surgery, Northwestern University, Feinberg School of Medicine, Chicago, IL, USA
}

Background Achieving symmetry is a key goal in breast reconstruction. Anatomically shaped tabbed expanders are a new tool in the armamentarium of the breast reconstruction surgeon. Suture tabs allow for full control over the expander position and thus inframammary fold position, and, in theory, tabbed expanders mitigate many factors responsible for poor symmetry. The impact of a tabbed expander on breast symmetry, however, has not been formally reported. This study aims to evaluate breast symmetry following expander-implant reconstruction using tabbed and non-tabbed tissue expanders.

Methods A chart review was performed of 188 consecutive expander-implant reconstructions that met the inclusion criteria of adequate follow-up data and postoperative photographs. Demographic, oncologic, postoperative complication, and photographic data was obtained for each patient. The photographic data was scored using a 4-point scale assessing breast symmetry by three blinded, independent reviewers.

Results Of the 188 patients, 74 underwent reconstruction with tabbed expanders and 114 with non-tabbed expanders. The tabbed cohort had significantly higher symmetry scores than the non-tabbed cohort $(2.82 / 4 \pm 0.86$ vs. $2.55 / 4 \pm 0.92, \mathrm{P}=0.034)$.

Conclusions The use of tabbed tissue expanders improves breast symmetry in tissue expanderimplant-based breast reconstruction. Fixation of the expander to the chest wall allows for more precise control over its location and counteracts the day-to-day translational forces that may influence the shape and location of the expander pocket, mitigating many factors responsible for breast asymmetry.

Keywords Breast / Mammaplasty / Tissue expansion devices / Outcome assessment (health care)
Correspondence: John YS Kim

Division of Plastic and Reconstructive

Surgery, Northwestern University,

Feinberg School of Medicine, $675 \mathrm{~N}$

St. Clair St., 19-250, Chicago,

IL 60611, USA

Tel: +1-312-695-6022

Fax: +1-312-695-5672

E-mail: jokim@nmh.org

Received: 14 Jun $2013 \bullet$ Revised: 7 Aug $2013 \bullet$ Accepted: 8 Aug 2013

pISSN: 2234-6163 • elSSN: 2234-6171 • http://dx.doi.org/10.5999/aps.2014.41.1.57 • Arch Plast Surg 2014;41:57-62

John YS Kim receives research funding from the Musculoskeletal Transplant Foundation.

No potential conflict of interest relevant to this article was reported.

\section{INTRODUCTION}

The rate of mastectomy for breast cancer has remained unchanged at approximately $35 \%$, despite advances in breast conservation therapy [1]. In 2012, over 91,000 breast reconstructions were performed by surgeons affiliated with the American Society of Plastic Surgeons, of which 70\% were tissue expander (TE)- implant reconstructions and $21 \%$ were autologous tissue reconstructions [2]. Both immediate and delayed breast reconstructions are known to provide substantial psychosocial benefits for mastectomy patients $[3,4]$. While many aspects of the reconstructive process are important to patient-reported outcomes, breast symmetry is one such significant factor that drives overall patient satisfaction [5-7]. 
Anatomically shaped, tabbed expanders are gaining popularity for TE-based breast reconstruction [8]. The suture tabs allow for predictable and fixed positioning of the expander in relation to preoperative measurements. The tabs also give the surgeon full control over various symmetry factors including the position of the inframammary fold (IMF), expander height, and its medial-lateral translation [8]. Several metrics have been used to report breast symmetry, most often utilizing a four-point scale to assess factors such as inframammary fold position, size, and shape [9-13]. However, to our knowledge, symmetry outcomes have never been compared between tabbed and non-tabbed TE-based breast reconstruction.

We hypothesized that the superior control over an expander's intraoperative and postoperative position would translate into improved breast symmetry and thus an improved aesthetic outcome. The aim of the current study was to determine whether the use of tabbed expanders resulted in improved symmetry scores in comparison to non-tabbed expanders in a single surgeon series of 188 expander-implant reconstructions.

\section{METHODS}

A retrospective chart review of consecutive expander-implant breast reconstructions by the senior author between 2004 and 2012 was performed with Institutional Review Board approval. Demographic, oncologic, operative, and photographic data were obtained for each patient. Criteria for inclusion were a complete demographic and oncologic history and photographic followup at least 30 days after completion of the expander-implant exchange.

All of the reconstructions were carried out by the senior author (J.K.). After the mastectomy, the lower border of the pectoralis was disinserted and a tissue expander was placed in the submuscular space. Tabbed tissue expanders (Mentor CPX3 Medium Height Expander) were sutured to the chest wall using 2-0 Maxon or polydioxanone (PDS). Non-tabbed tissue expanders included the Mentor CPX2 medium height tissue expander and Allergan Natrelle 133-MV tissue expander. Once complete expander coverage had been obtained, 7-mm clot stop drains (Axiom, Torrance, CA, USA) were placed in the inferior space between the mastectomy flap and the expander, and in the axillary and superior subcutaneous planes. The tissue expander was judiciously inflated with saline in proportion to the degree of skin excess. Postoperatively, the drains were maintained in place until the output was less than $30 \mathrm{~mL}$ per 24 hours. Routine perioperative antibiotic prophylaxis was given. After the incisions healed, the patient underwent serial tissue expander expansions, with intervals and volumes of serial tissue expan- sions determined on a per patient basis. Upon completing tissue expansions and adjuvant therapy, second-stage reconstruction with tissue expander-to-implant exchange was performed. The permanent implants were moderate plus or high profile Mentor smooth, round, saline breast implants or Mentor MemoryGel smooth, round breast implants.

The demographic and oncologic variables included the following: age, body mass index (BMI), active smoking status, diabetes, hypertension, post-mastectomy radiation, prior history of radiation, adjuvant chemotherapy, acellular dermal matrix use, and history of unilateral or bilateral reconstruction. The operative characteristics included tabbed versus non-tabbed tissue expanders, intraoperative fill, final tissue expander fill, and permanent implant volume. Photographic follow-up refers to the number of weeks from the expander-implant exchange to the date of the photograph.

For the primary endpoint of this study, three blinded members of the Northwestern Feinberg School of Medicine Division of Plastic and Reconstructive Surgery were asked to independently rate anterior photographs of the patients' breasts using a 4-point scale (1-4) with respect to symmetry, with 1 being unacceptable symmetry and 4 being excellent symmetry. The scale was based on previous studies of breast symmetry $[10,14]$. Raters were instructed to consider symmetry of the breast contour, mound volume, and inframammary fold placement. Additional surgical procedures for improvement of symmetry and cosmesis following primary reconstruction were tracked as a secondary outcome.

The aesthetic scores of the tabbed and non-tabbed tissue expander cohorts were compared with a Student's t-test. Satisfactory inter-rater reliability for the aesthetic scoring was determined by calculating Fleiss's kappa. All of the other variables were compared with Student's t-tests, Mann-Whitney U-tests, and either chi-squared or Fischer's exact tests, as appropriate. All analyses were performed with SPSS ver. 20.0 (IBM Corp., Armonk, NY, USA).

\section{RESULTS}

Of the 188 patients meeting the inclusion criteria, 74 underwent reconstruction with anatomically shaped tabbed expanders and 114 underwent reconstruction with traditional expanders without suture tabs. The mean follow-up at the time of the photograph was 30 weeks in the tabbed cohort and 35 weeks in the non-tabbed cohort $(\mathrm{P}=0.098)$. A photograph of a tabbed expander is shown in Fig. 1.

Within the tabbed cohort, $48.7 \%$ of the cases were unilateral compared to $52.6 \%$ of the non-tabbed cases $(\mathrm{P}=0.594)$. Acel- 


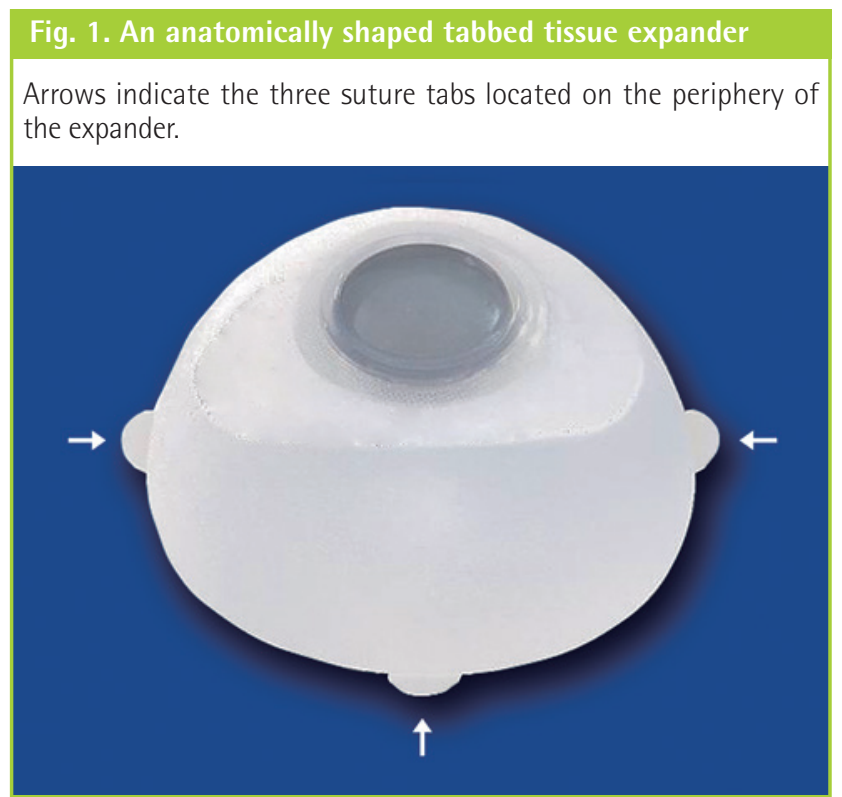

lular dermal matrix use also did not differ between the cohorts ( $44.6 \%$ tabbed vs. $57.0 \%$ non-tabbed, $\mathrm{P}=0.096$ ). The cohorts were similar with respect to age (49.1 years vs. 50.9 years, $\mathrm{P}=$ $0.309)$ and BMI $\left(27.3 \mathrm{~kg} / \mathrm{m}^{2}\right.$ vs. $\left.27.4 \mathrm{~kg} / \mathrm{m}^{2}, \mathrm{P}=0.914\right)$, and did not differ in the prevalence of hypertension (18.9\% in tabbed cohort vs. $20.2 \%, \mathrm{P}=0.832$ ) or proportion of active smokers ( $4.1 \%$ vs. $6.7 \%, \mathrm{P}=0.169$ ). More patients in the tabbed expander group had diabetes $(6.8 \%$ vs. $0.9 \%, \mathrm{P}=0.036)$. Preoperative and adjuvant radiation rates did not differ between the cohorts (5.4\% tabbed vs. $1.8 \%$ non-tabbed, $\mathrm{P}=0.214$ and $17.6 \%$ tabbed vs. $29.0 \%$ non-tabbed, $\mathrm{P}=0.084$, respectively). However, more patients in the non-tabbed expander group received adjuvant chemotherapy ( $53.5 \%$ non-tabbed vs. $35.1 \%$ tabbed, $\mathrm{P}=0.017$ ). Although there was no difference in intraoperative expander fill between the cohorts ( $236 \mathrm{~mL}$ vs. $221 \mathrm{~mL}, \mathrm{P}=0.580)$, the tabbed expander cohort experienced larger final tissue expander volumes ( $479 \mathrm{~mL}$ vs. $407 \mathrm{~mL}, \mathrm{P}=0.014)$ and larger permanent implant volumes ( $490 \mathrm{~mL}$ vs. $421 \mathrm{~mL}, \mathrm{P}=0.007$ ). A summary of demographic, oncologic, and surgical information is presented in Table 1.

The tabbed expander cohort demonstrated significantly higher symmetry scores than the non-tabbed expander cohort (2.82/4 \pm 0.86 vs. $2.55 / 4 \pm 0.92, \mathrm{P}=0.034)$. There were no significant differences in the symmetry score upon stratifying by unilateral $(2.48 / 4 \pm 0.77$ tabbed vs. $2.27 / 4 \pm 0.82, \mathrm{P}=0.206)$ and bilateral $(3.15 / 4 \pm 0.74$ vs. $2.86 / 4 \pm 0.89, \mathrm{P}=0.103)$ reconstruction. The average number of additional surgical procedures to improve symmetry and cosmesis following reconstruction did not differ significantly between the two cohorts $(1.12 / 4 \pm 1.12$ tabbed vs. $0.89 / 4 \pm 0.99, \mathrm{P}=1.39)$. The aesthetic scores for the tabbed and non-tabbed tissue expander cohorts
Table 1. Demographic and oncologic characteristic

\begin{tabular}{|lccc|}
\hline Characteristics & $\begin{array}{c}\text { Tabbed } \\
(\mathbf{n}=\mathbf{7 4})\end{array}$ & $\begin{array}{c}\text { Non-tabbed } \\
(\mathbf{n}=114)\end{array}$ & P-value ${ }^{\text {a) }}$ \\
\hline Age $(\mathrm{yr})$ & $49.1 \pm 11.8$ & $50.9 \pm 11.8$ & 0.309 \\
Body mass index $\left(\mathrm{kg} / \mathrm{m}^{2}\right)$ & $27.3 \pm 5.6$ & $27.4 \pm 6.0$ & 0.914 \\
Active smoker $(\%)$ & $3(4.05)$ & $11(9.65)$ & 0.169 \\
Diabetes (\%) & $5(6.76)$ & $1(0.88)$ & 0.036 \\
Hypertension (\%) & $14(18.92)$ & $23(20.18)$ & 0.832 \\
Preoperative radiation (\%) & $4(5.41)$ & $2(1.75)$ & 0.214 \\
Adjuvant radiation (\%) & $13(17.57)$ & $33(28.95)$ & 0.084 \\
Adjuvant chemotherapy (\%) & $26(35.14)$ & $61(53.51)$ & 0.017 \\
Acellular dermal matrix use (\%) & $33(44.59)$ & $65(57.02)$ & 0.096 \\
Unilateral reconstruction (\%) & $36(48.65)$ & $60(52.63)$ & 0.594 \\
Intraoperative fill (mL) & $236 \pm 217$ & $221 \pm 151$ & 0.580 \\
Final expander volume (mL) & $479 \pm 180$ & $407 \pm 18$ & 0.014 \\
Permanent implant volume (mL) & $490 \pm 163$ & $421 \pm 136$ & 0.007 \\
Photo follow-up time (wk) & $29.7 \pm 17.8$ & $35.1 \pm 24.2$ & 0.098 \\
\hline Values are presented as mean \pm standard deviation or number (\%). Bolded \\
characteristics are significant at the 0.05 level. & \\
a) Chi-squared and t-tests for categorical and continuous variables, respectively. \\
\hline
\end{tabular}

are summarized in Table 2. These analyses were repeated including only those patients with a follow-up of 6 months or greater at the time of the photograph. The tabbed expander cohort $(\mathrm{n}=58)$ again demonstrated a significantly greater symmetry score than the non-tabbed expander cohort $(\mathrm{n}=79)$ (2.83/4 \pm 0.89 tabbed vs. $2.49 \pm 0.92, \mathrm{P}=0.032)$. The average number of additional surgical procedures following reconstruction was again not significant between the cohorts $(1.09 \pm 1.08$ tabbed vs. $0.96 \pm 1.07, \mathrm{P}=0.504$ ).

\section{DISCUSSION}

Tissue expanders used in breast reconstruction have evolved dramatically since their introduction $[15,16]$. Anatomically shaped tabbed expanders (Fig. 1) represent one of the latest innovations within the field. These expanders feature an integrated port and three circumferentially positioned suture tabs, which allow for precise placement of the expander within the sub-pectoral pocket [8]. In addition, these expanders are anatomically shaped with a larger inferior pole and a slope similar to a natural ptotic breast. Control over lower pole expansion and the ability to fix the implant exactly on the chest wall may mitigate factors responsible for poor breast reconstruction symmetry. However, to our knowledge, there are no published reports evaluating breast symmetry following reconstruction with tabbed expanders.

In this single-surgeon perspective, patients were evaluated by three independent raters, on a four-point scale $[10,14]$, with high inter-rater reliability (Kappa statistic 0.75 ). Overall, reconstructions using tabbed expanders demonstrated superior symmetry following expander-to-implant exchange when compared 


\begin{tabular}{|c|c|c|c|}
\hline Characteristics & $\begin{array}{l}\text { Tabbed } \\
(n=74)\end{array}$ & $\begin{array}{l}\text { Non-tabbed } \\
(n=114)\end{array}$ & P-value \\
\hline \multicolumn{4}{|l|}{ Symmetry score ${ }^{\text {b) }}$} \\
\hline All reconstructions & $2.82 \pm 0.86$ & $2.55 \pm 0.92$ & 0.034 \\
\hline Unilateral reconstructions & $2.48 \pm 0.77$ & $2.27 \pm 0.82$ & 0.206 \\
\hline Bilateral reconstructions & $3.15 \pm 0.74$ & $2.86 \pm 0.89$ & 0.103 \\
\hline Additional symmetry procedures & $1.12 \pm 1.12$ & $0.89 \pm 0.99$ & 0.139 \\
\hline
\end{tabular}

to those using non-tabbed expanders (2.82/4 for tabbed vs. $2.55 / 4$ for non-tabbed, $\mathrm{P}=0.034$ ) (Table 2 ). This trend continued to remain significant after limiting the analyses to only those patients with a follow-up of at least 6 months at the time of the photograph $(2.83 / 4 \pm 0.89$ tabbed vs. $2.49 \pm 0.92)(\mathrm{P}=0.032)$. We hypothesized that fixation of expanders to the chest wall via suture tabs allows for more precise placement of expanders with respect to pre-surgical vertical and horizontal positioning measurements, and for resistance of translational forces exerted on TEs by daily activities, capsular contraction, overlying soft tissue tension, and body habitus.

The present study is believed to be the first report of symmetry scores following tabbed expander-to-implant reconstructions; however, we previously showed that when used in conjunction with latissimus flaps for TE revisions, the tabs facilitated expander placement with the patient remaining in the lateral decubitus position [8]. Eliminating the traditional position change reduced the surgical time by 100 minutes, and aesthetic scores in the lateral decubitus position did not differ from those achieved with traditional supine repositioning. These findings highlight the improved control over expander pocket placement and reduction in expander migration that tabbed expanders afford us and suggest a role for tabbed expanders in other types of breast reconstruction.

Achieving symmetry in bilateral breast reconstruction is often technically easier than in unilateral reconstructions [17]. By manipulating both breasts, surgeons can overcome intrinsic structural and anatomic limitations in the positioning of the pectoralis origin at the thoracic wall, positioning of the IMF, breast mound height, and medial-lateral translation. In this study, we found superior symmetry scores in bilateral reconstructions compared to unilateral reconstructions (bilateral 2.98/4 vs. unilateral 2.35/4, $\mathrm{P}<0.001$ ) (Table 3). We hypothesized that the use of two TEs also predisposes patients to a greater risk of migration and asymmetry than one TE. Securing the TEs to the chest wall via suture tabs should prevent these asymmetries and therefore improve symmetry scores within the bilateral cohort. In our study, tabbed expanders indeed demonstrated a trend
Table 3. Aesthetic outcomes in unilateral and bilateral reconstructions

\begin{tabular}{|lccc|}
\hline & $\begin{array}{c}\text { Unilateral } \\
(\mathrm{n}=74)\end{array}$ & $\begin{array}{l}\text { Bilateral } \\
(\mathrm{n}=114)\end{array}$ & P-value $^{\text {a) }}$ \\
\hline Symmetry score ${ }^{\text {a) }}$ & $2.35 \pm 0.80$ & $2.98 \pm 0.84$ & $<0.001$ \\
\hline $\begin{array}{l}\text { Bolded characteristics are significant at the } 0.05 \text { level. } \\
\text { a'T-test; bi Kappa statistic, } 0.749 .\end{array}$ & & \\
\hline
\end{tabular}

toward greater symmetry scores than non-tabbed expanders in the bilateral reconstruction group (3.15/4 tabbed vs. 2.86/4 non-tabbed, $\mathrm{P}=0.103$ ); however, we suspect that this sub-group analysis decreased our power to identify a significant difference. Larger studies of tabbed versus non-tabbed expanders for symmetry achievement are warranted in bilateral TE-based breast reconstructions.

Achieving symmetry in unilateral breast reconstruction can be more technically demanding because the native breast tissue has a gentler slope and more ptotic appearance than a breast reconstructed with a round implant [17]. The use of tabs to secure the TE in place may provide the best chance of matching the native breast, especially in the position of the IMF. For these reasons the lower symmetry scores for unilateral reconstruction demonstrated here were expected (unilateral 2.35 vs. bilateral $2.98, \mathrm{P}<0.001$, Table 3 ). The suture tabs on the TEs presumably make a tabbed expander more likely to remain where the surgeon has placed it, resulting in greater symmetry compared to non-tabbed expanders in unilateral reconstructions. This trend was also observed in the present study (tabbed 2.48/4 vs. non-tabbed 2.27/4, $\mathrm{P}=0.206$ ); however, this subgroup analysis again did not have a large enough sample size to demonstrate statistical significance. In addition to gentle contours and symmetric IMFs, the native breast is usually more ptotic, and makes an artificial implant stand out against a natural contralateral breast. For these reasons, a subsequent post-reconstruction symmetry procedure is often necessary [17]. Further efforts to improve unilateral reconstructions led to the evolution in use and type of TE available $[16,18]$, including the development of anatomically-shaped TEs [19-21], like those used in the present study, as well as anatomically shaped implants. Breast implants that are anatomically-shaped have been used for over a decade in Europe and South America [19,20,22] and were recently approved for use in the United States [23]. A corresponding well-suited pocket could be created for such implants by anatomically-shaped TEs, which might optimize unilateral breast reconstruction. Further research into optimizing unilateral breast reconstruction is needed.

A number of strategies have been developed to improve breast symmetry following reconstruction, often involving multiple 
surgical revisions of the reconstructed breast. These additional procedures incur greater costs for the patient, increase the risk of poor outcomes, and delay the completion of reconstruction $[17,24,25]$. Despite improving symmetry scores, the use of tabbed expanders, surprisingly, did not yield a significant difference in the number of elective revision procedures (tabbed 1.12 vs. non-tabbed $0.89, \mathrm{P}=0.139$ ) (Table 2). Nonetheless, these additional procedures could potentially confound our results, and larger or prospective randomized studies are warranted to better moderate the effect of this confounding factor on breast symmetry scores. Furthermore, while it is reasonable to infer that a patient's general dissatisfaction with the cosmetic outcomes of the reconstruction contributed to their decision to undergo revisions, it was not possible to determine the extent to which poor symmetry contributed to that decision. Future studies assessing patient satisfaction following reconstruction with a tabbed expander can more clearly shed light on the patient's perception of the aesthetic outcomes and their motivations for any surgical revisions.

Though this study faces a number of limitations, most importantly the constrained statistical power associated with an emerging cohort, we are optimistic that our group's growing experience with tabbed expanders will yield future insights on this novel technique [8]. In addition to previously mentioned confounders, the senior surgeon's experience with breast reconstruction, with or without tabbed expanders, undoubtedly improves over the years, once again, potentially confounding our outcomes. The employment of regression and other sophisticated statistical methods with a larger cohort will help to mitigate these and other potential confounders that limit the current analysis. Furthermore, although the scoring scale used in this study achieved satisfactory inter-rater reliability and sensitivity to discern differences in symmetry, longer photographic follow-up, additional lateral or oblique-lateral views, and computer assisted analysis of photographic data in future studies will enable precise topographic mapping and the identification of irregularities in symmetry and a number of other cosmetic parameters [9].

In a continuously evolving field like that of breast reconstruction, a more thorough understanding of cosmetic outcomes is crucial to making well-informed, evidence-based decisions in patient care. The ability to fix the expander on the chest wall allows for predictable positioning of the pocket in relation to preoperative measurements and mitigates factors responsible for poor breast reconstruction symmetry. This study provides important preliminary evidence of an improvement in breast symmetry following reconstruction with an anatomically shaped tabbed expander. Achieving favorable cosmetic outcomes, including symmetry, poses a significant challenge to reconstruc- tive surgeons and the potential for tabbed expanders to improve these outcomes safely warrants further investigation.

\section{REFERENCES}

1. Baxter NN, Virnig BA, Durham SB, et al. Trends in the treatment of ductal carcinoma in situ of the breast. J Natl Cancer Inst 2004;96:443-8.

2. American Society of Plastic Surgeons. 2012 Plastic surgery statistics report [Internet]. Arlington Heights; American Society of Plastic Surgeons: c2013 [cited 2013 May 26]. Available from: http://www.plasticsurgery.org/Documents/ news-resources/statistics/2012-Plastic-Surgery-Statistics/ reconstructive-surgery-demographics.pdf.

3. Wilkins EG, Cederna PS, Lowery JC, et al. Prospective analysis of psychosocial outcomes in breast reconstruction: oneyear postoperative results from the Michigan Breast Reconstruction Outcome Study. Plast Reconstr Surg 2000;106: 1014-25.

4. Pusic AL, Klassen AF, Scott AM, et al. Development of a new patient-reported outcome measure for breast surgery: the BREAST-Q. Plast Reconstr Surg 2009;124:345-53.

5. Pusic AL, Chen CM, Cano S, et al. Measuring quality of life in cosmetic and reconstructive breast surgery: a systematic review of patient-reported outcomes instruments. Plast Reconstr Surg 2007;120:823-37.

6. Bezuhly M, Bucholtz J, Sigurdson L. Less scarring or more symmetry? Reconstruction following metachronous bilateral breast cancer. J Plast Reconstr Aesthet Surg 2012;65: 1595-7.

7. Craft RO, Colakoglu S, Curtis MS, et al. Patient satisfaction in unilateral and bilateral breast reconstruction [outcomes article]. Plast Reconstr Surg 2011;127:1417-24.

8. Gust MJ, Nguyen KT, Hirsch EM, et al. Use of the tabbed expander in latissimus dorsi breast reconstruction. J Plast Surg Hand Surg 2013;47:126-9.

9. Szychta P, Butterworth M, Dixon M, et al. Breast reconstruction with the denervated latissimus dorsi musculocutaneous flap. Breast 2013;22:667-72.

10. Henseler H, Smith J, Bowman A, et al. Subjective versus objective assessment of breast reconstruction. J Plast Reconstr Aesthet Surg 2013;66:634-9.

11. Fortin AJ, Cheang M, Latosinsky S. Cosmetic outcomes following breast conservation therapy: in search of a reliable scale. Breast Cancer Res Treat 2006;100:65-70.

12. Yesilada AK, Sevim KZ, Sirvan SS, et al. Our surgical approach to treatment of congenital, developmental, and acquired breast asymmetries: a review of 30 cases. Aesthetic 
Plast Surg 2013;37:77-87.

13. Kasielska A, Antoszewski B. Surgical management of gynecomastia: an outcome analysis. Ann Plast Surg 2013;71:471-5.

14. Harris JR, Levene MB, Svensson G, et al. Analysis of cosmetic results following primary radiation therapy for stages I and II carcinoma of the breast. Int J Radiat Oncol Biol Phys 1979;5:257-61.

15. Radovan C. Breast reconstruction after mastectomy using the temporary expander. Plast Reconstr Surg 1982;69:195-208.

16. Yanko-Arzi R, Cohen MJ, Braunstein R, et al. Breast reconstruction: complication rate and tissue expander type. Aesthetic Plast Surg 2009;33:489-96.

17. Losken A, Carlson GW, Bostwick J 3rd, et al. Trends in unilateral breast reconstruction and management of the contralateral breast: the Emory experience. Plast Reconstr Surg 2002;110:89-97.

18. Buck DW 2nd, Heyer K, DiBardino D, et al. Acellular dermis-assisted breast reconstruction with the use of crescentric tissue expansion: a functional cosmetic analysis of 40 consecutive patients. Aesthet Surg J 2010;30:194-200.

19. McGeorge DD, Mahdi S, Tsekouras A. Breast reconstruction with anatomical expanders and implants: our early experience. Br J Plast Surg 1996;49:352-7.
20. Gui GP, Tan SM, Faliakou EC, et al. Immediate breast reconstruction using biodimensional anatomical permanent expander implants: a prospective analysis of outcome and patient satisfaction. Plast Reconstr Surg 2003;111:125-38.

21. Mahdi S, Jones T, Nicklin S, et al. Expandable anatomical implants in breast reconstructions: a prospective study. $\mathrm{Br} \mathrm{J}$ Plast Surg 1998;51:425-30.

22. Maxwell GP, Falcone PA. Eighty-four consecutive breast reconstructions using a textured silicone tissue expander. Plast Reconstr Surg 1992;89:1022-34.

23. FDA. Natrelle 410 highly cohesive anatomically shaped silicone-filled breast implant-P040046 [Internet]. Silver Spring, MD: U.S. Food and Drug Adimistration; c2013 [cited 2013 May 26]. Available from: http://www.fda.gov/ MedicalDevices/ProductsandMedicalProcedures/DeviceApprovalsandClearances/Recently-ApprovedDevices / ucm343639.htm.

24. Beahm EK, Walton RL. Revision in autologous breast reconstruction: principles and approach. Clin Plast Surg 2007;34:139-62.

25. Stevenson TR, Goldstein JA. TRAM flap breast reconstruction and contralateral reduction or mastopexy. Plast Reconstr Surg 1993;92:228-33. 